\title{
Nous avons tous une histoire
}

\author{
par Patrick Fitch
}

$\mathrm{V}$ ous lirez ce texte au printemps, mais, au moment d'écrire ces lignes, le temps des fêtes commence tout juste à prendre de la vigueur. Les assauts publicitaires ont atteint leur paroxysme et l'on se sent légèrement dépassé. Malgré cette cacophonie, des récits de bienveillance se font entendre. Ceux-ci se dressent en rempart contre l'enchainement constant de mauvaises nouvelles : que l'on parle d'écrasement d'avion, de guerre, de terrorisme ou - un sujet plus près de notre profession - de l'épidémie de surdose de fentanyl. Peu importe comment vous vous informez de ce qui se passe dans le monde - par la télévision, les journaux ou les médias sociaux -, le temps des fêtes semble nous apporter une vague d'histoires optimistes qui donnent chaud au cœur. Je ne sais pas si ce moment de l'année fait ressortir ce qu'il y a de meilleur chez les gens ou si les médias accordent plus d'attention à ce genre d'histoires durant cette période, mais, dans mon cas, j’apprécie le répit de la morosité dont on nous abreuve habituellement.

Voici où je veux en venir : je crois que ces histoires doivent être racontées. C'est en racontant des histoires, des récits que nous communiquons les uns avec les autres. Penser à cela m'a aussi fait réfléchir à notre manière de communiquer avec les autres dans notre milieu de travail et nos pratiques de la pharmacie.

En fait, raconter des histoires est un élément essentiel de notre façon de pratiquer. Lorsqu'on participe aux tournées médicales, nous commençons souvent par l'histoire de chaque patient et comment leurs soins nous ont été confiés. L'histoire de chaque patient est unique et, à titre de professionnels de la santé, nous avons une influence importante sur elle.

On peut raconter des récits de bien des manières. Combien de fois, en compagnie de collègues, une personne a-t-elle dit « j’ai ce patient qui... »? La plupart du temps, nous racontons l'histoire du patient pour obtenir des conseils de collègues ou pour communiquer une nouvelle connaissance que nous avons acquise. En effet, la plupart d'entre nous apprennent et retiennent plus facilement avec des histoires qu'avec une énumération aride de faits.
Ce sur quoi nous devons travailler, c'est notre hésitation à raconter les histoires qui célèbrent nos réussites. Soyons francs : le domaine de la santé est rempli d'histoires à propos « de mauvaises nouvelles » dans lesquelles le patient meurt ou connaît de mauvais résultats ou est victime de systèmes de soutien défaillants qui rendent difficile la maîtrise de maladies chroniques. Mais il y a aussi beaucoup d'histoires au sujet de "bonnes nouvelles ". Nous connaissons tous des patients à l'article de la mort qui se sont malgré tout rétablis. Il y a aussi ceux qui ont obtenu de meilleurs résultats que ce à quoi l'on s'attendait grâce à nos soins. Je crois qu'en tant que pharmaciens, nous ne prenons généralement pas le temps nécessaire pour souligner nos réussites et cela s'applique autant à nous individuellement comme membres d'une équipe de soins qu'à notre profession dans son ensemble.

Même s'il est un peu tard pour les résolutions du Nouvel An, je vous invite tous à prendre un moment cette année pour vous raconter des histoires entre collègues. Il faut faire connaître tout le bon travail que nous faisons en tant que profession et en tant que professionnels de la santé dévoués. En ce sens, le thème du 43e séminaire de Banff des sections de l'Ouest de la SCPH (qui a eu lieu du 17 au 19 mars 2017) était «Le récit : communiquer grâce à nos expériences ». Les organisateurs ont invité des experts du récit pour inspirer, stimuler et éclairer.

Enfin, pour paraphraser l'auteur Philip Pullman, " les histoires sont ce dont nous avons le plus besoin en ce monde.»

[Traduction par l'éditeur]

Patrick Fitch, BSP, ACPR, est président désigné et agent de liaison interne pour la Société canadienne des pharmaciens d'hôpitaux. 